\title{
3d Ear Shape Biometric Recognition by Using Ica Alorithm
}

\author{
Isha Das, Srinivasa Raja G \\ Sree Sastha Institute of Engineering and Technology,Chennai-600123,Tamilnadu,India.
}

\begin{abstract}
Biometrics are physical or behavioral characteristics that can be used for human identification. In this dissertation, we propose the ear as a biometric and investigate its potential with both 2D and 3D data.This work is the largest experimental investigation of ear biometrics to date. The data set used for this experiments contains 50 persons, each with images acquired on at least two different dates. Approaches considered include a ICP ("eigen-ear") approach with 2D intensity images and range images, a Hausdorff matching of edges from range images, and an ICA-based approach to matching the 3D data. This experimental results demonstrate that the ICA-based approach outperforms the other approaches at a statistically significant level. Furthermore, this will develop the first fully automated biometric system using 3D ear shape. It is a complete automatic system starting with a raw 3D image, through automated segmentation of the ear, and 3D shape matching for recognition. The automatic system achieves a rank-one recognition rate of $97.6 \%$ on a 50 -subject dataset. This algorithm shows good scalability of recognition rate with size of dataset. The results suggest a strong potential for 3D ear shape as a biometric. However, some people have ears with recognizably different shapes. Experimental results with partial ear shape suggest that minor hair covering does not affect the performance substantially, but large hair covering will certainly reduce the recognition rate. This suggests that even in circumstances where the complete ear shape cannot be captured, partial shape has potential for recognition. This lends support for using ear shape as a biometric.
\end{abstract}

Index Terms: Iterative Closet Point (ICP), Independent Component Analysis (ICA)

\section{INTRODUCTION}

Biometrics is the study of measuring physical or behavioral characteristics of a person to verify orrecognize his or her identity.Public afety and national security enhance the needs for biometric techniques, which are among the most secure and accurate authentication tools. Ear biometric is invariant with age (or) time, It is not prone to accumulative errors. In this the footage can be taken by using CCTV (or) incorporated into cell phones and for the suspicious person's identification can be done easily.Ears are remarkably consistent. Unlike faces, they do not change shape with different expressions or age, and remain fixed in the middle of the side of the head against a predictable background. Hair is a problem, but that might be solved by using infrared images.

\section{A .Types of ear Biometric}

There are at least three methods for ear identification:

(1) taking a photo of an ear,

(2) taking "earmarks" by pushing ear against a flat glass and

(3) taking thermogram pictures of the ear.

The most interesting parts of the ear are the outer ear and ear lope, but the whole ear structure and shape is used. Taking photo of the ear is the most commonly used method in research. The photo is taken and it is combined with previous taken photos for identifying a person. The earmarks are used mainly in crime solving. Even though some judgments are made based on the earmarks, currently they are not accepted in courts. The thermogram pictures could be one solution for solving the problem with e.g. hair of hat.

\section{B. Anatomy of External Ear}

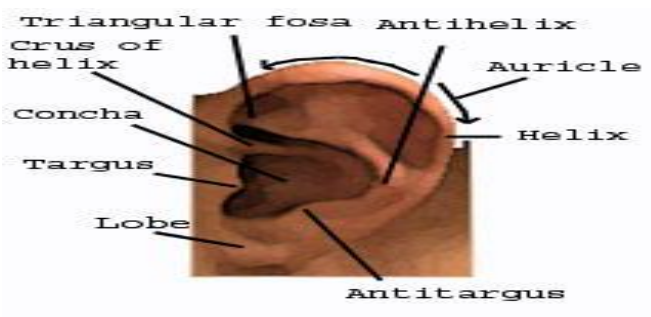


II. PROPOSED APPROACH

Fig.1 Anatomy of External Ear

The 3-D ear recognition has been done here by using ICA (Independent Component Analysis), before the other algorithms are used like ICP (Iterative closet point) algorithm and PCA(Principal Component Analysis).

\section{A. Ica (Independent Component Analysis)}

It is a computational method for separating a multivariate signal into additive subcomponents; this is the special case of blind source separation. This consist of separation of mixed signals, without aid of aid of information (or with very little information)of the source signal. It relies on the assumption that the source signals do not correlate with each other. It aims at extracting statically independent component, where classical techniques search for decorrelated components. Secondly the linear hypothesis for the mixture of components is not required. Example; cocktail party problem where speech signal separated from sample data consisting of people talking simultaneously in a room.

\section{B. 3d-Ear Shape Recognition Biometric Steps}

(i) Raw Image Extraction

The first stage is to extract the raw image for all individual from left profile by using camera or CCTV in its original form.

\section{(ii) Preprocessing}

In this stage the cropping has been done around the ear to produce a new image that is tightly cropped to minimize the appearance of background information in the image. All images are cropped to the same size. Conversion to gray scale is a pre-processing process that is done to all images and histogram equalization is performed on each image to compensate for lighting variations.

\section{(iii) Normalization}

Ear images have to be extracted and normalized with respect to scale and rotation, this is done by selecting two landmark in every image and transforming the image with respect to those landmarks to a new location. This transformation will result in getting the landmark points in all images to have the same distance between them and that orientation of connecting the two landmark points will be vertical in the image. The landmark points are selected manually for each image for example; the two landmark points can be Triangular Fossa and Incisure Intertragica in the ear. These location in the ear are selected because they prominently visible points in the ear.

\section{(iv) Training}

There are two phases in the identification phase: training and testing. In the training phase the eigenvalues and eigenvectors of the training set are extracted and the eigenvectors are chosen based on the top eigenvalues. Training set is a set of clean images without any duplicates. In the testing phase the algorithm is provided a set of known ears and faces and a set of unknown ears and faces as the probe set. The algorithm matches each probe to its possibly identity in the gallery. 


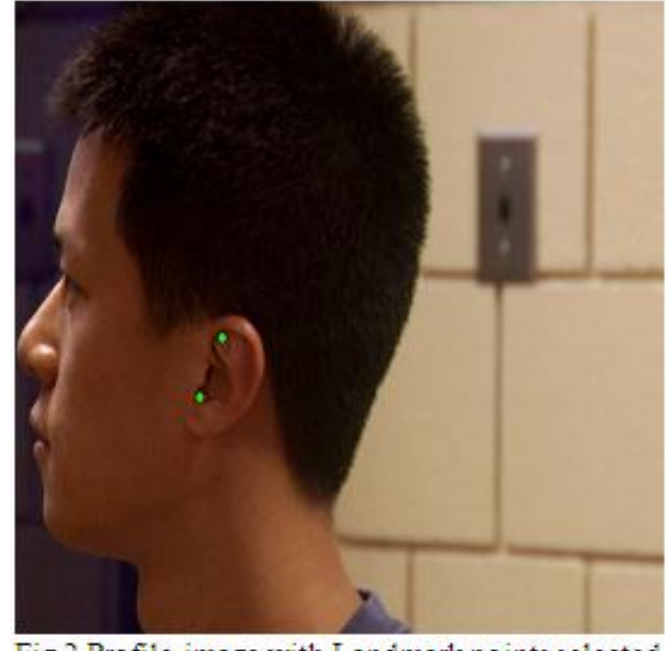

Fig.2 Profile image with L andmark points selected

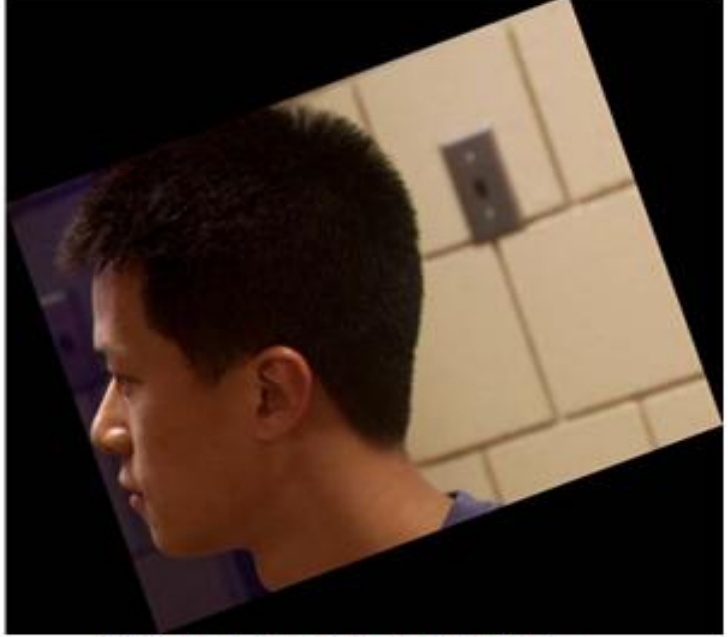

Fig.3 Image after scaling and rotation

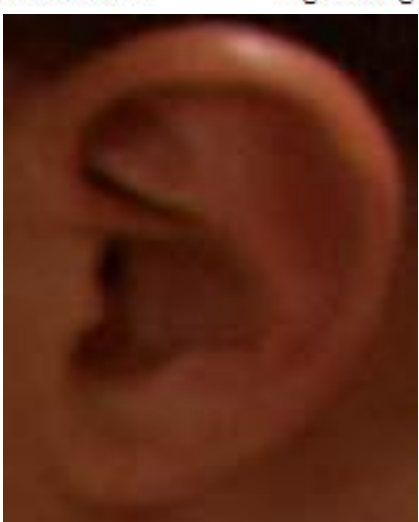

Fig.4 Ear image cropped

III.

SIMULATION AND RESULTS

\section{A. Details of Input Data and Output Result}

For input data number of ear images has been taken.

(1) All the ear images should be cropped in the same matrix size.

(2) Grey scaling of the cropped image has been done after that resize the image 300x300.

(3) Contour analysis has been accomplished then the mean and standard deviation is calculated.

\section{B. Output Result}

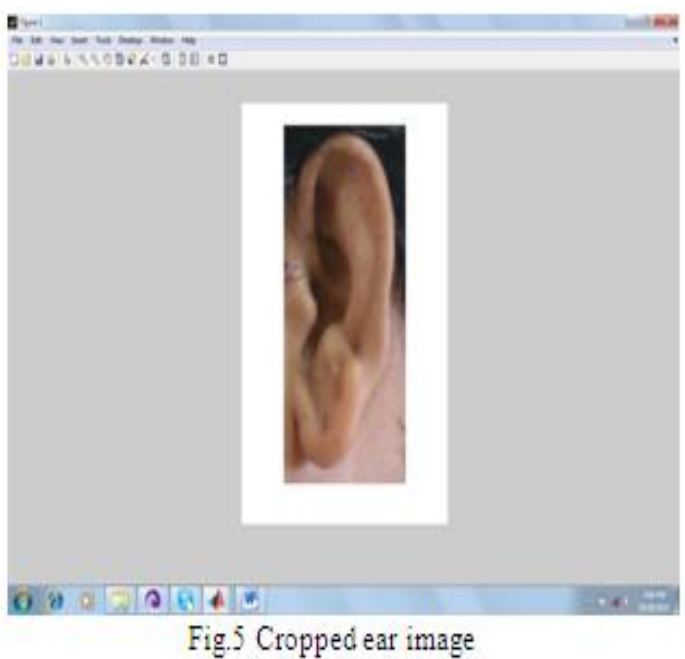

Fig.5 Cropped ear image

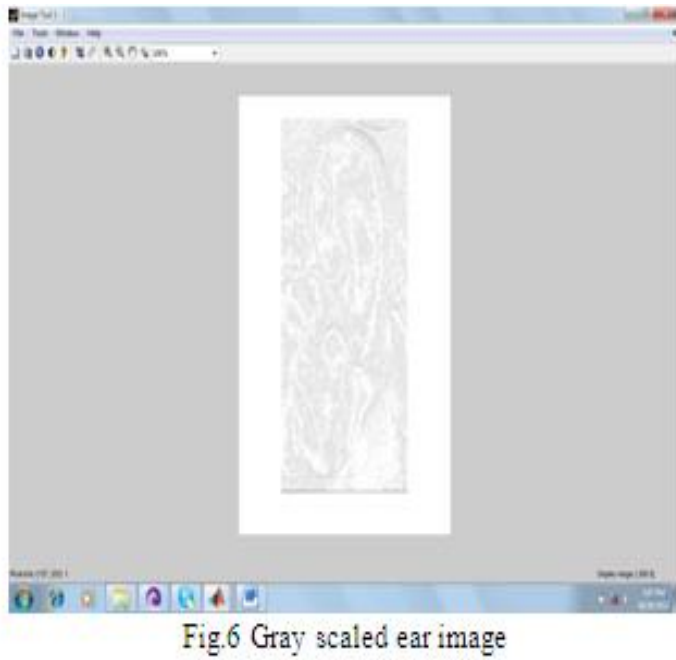

Fig.6 Gray scaled ear image 


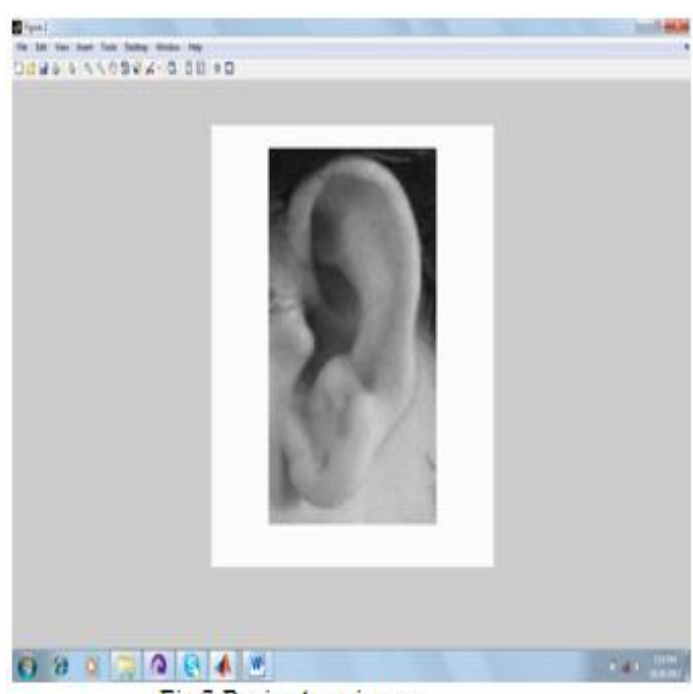

Fig.7 Resized ear image

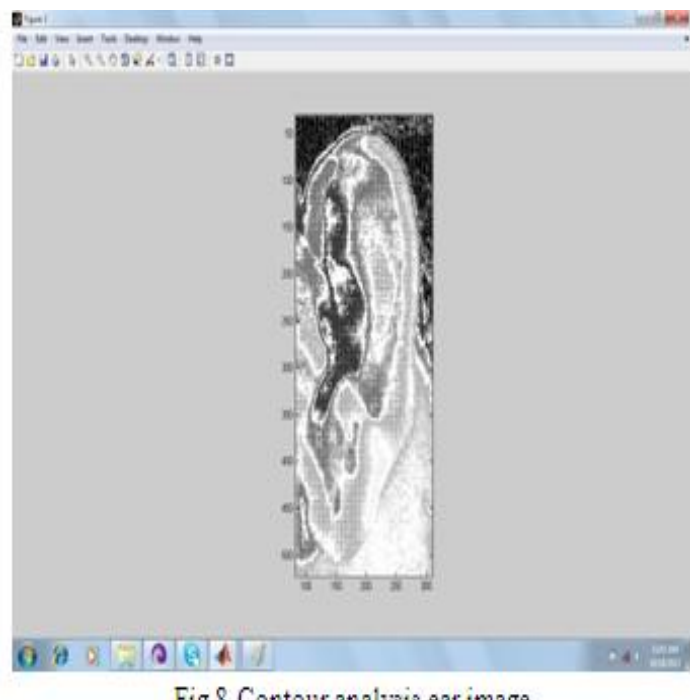

Fig.s Contour analysis ear image

C. Graph Representation of Mean and Standared Deviation

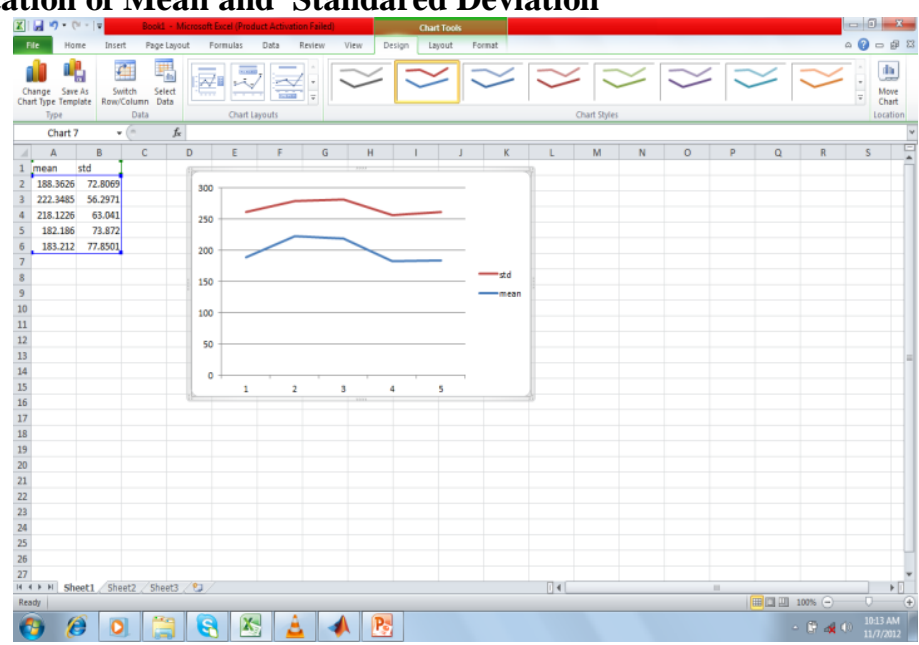

Fig.9 Graphical representation of mean and standard deviation

Table.1 Mean and Standard Deviation Values

\begin{tabular}{|c|c|}
\hline Mean & Standard Deviation \\
\hline 188.3626 & 72.8069 \\
\hline 222.3485 & 56.2971 \\
\hline 218.1226 & 63.041 \\
\hline 182.186 & 73.872 \\
\hline 183.212 & 77.8501 \\
\hline
\end{tabular}

\section{CONCLUSION}

A complete, automatic 3-D ear biometric system is presented by using the independent component analysis. The experimental results demonstrate the accuracy and efficiency of our novel 3-D ear shape matching approach. Moreover, the proposed approach achieves a significantly higher computational efficiency.

Future extensions of this work will include investigating the use of the proposed 3-D feature descriptors for general 3-D object detection, retrieval and recognition tasks. The FAR and FRR will be calculated for the number of samples .

\section{REFERENCES}

[1]. H. Chen and B. Bhanu, "Contour matching for 3-D ear recognition," in Proc. IEEE Workshop Applications Computer Vision, Jan. 2005, pp. 123-128. 
[2]. H. Chen and B. Bhanu, "Human ear recognition in 3D," IEEE Trans. Pattern Anal. Machine Intell., vol. 29, no. 4, pp. 718-737, Apr. 2007.

[3]. H. Chen and B. Bhanu, "Human ear recognition from side face range images," in Proc. Int. Conf. Pattern Recognition, Aug. 2004, pp. 574-577.

[4]. S. Islam, R. Davies, M. Bennamoun, and A. Mian, "Efficient detection and recognition of 3D ears," Int. J. Computer Vision, vol. 95, no. 1, pp. 52-73, 2011.

[5]. I.Kakadiaris, G.Passalis, G. Toderici,N.Murtuza,Y.Lu,K. N. , and T. Theoharis, "3D face recognition in the presence of facial expressions: An annotated deformable model approach," IEEE Trans. Pattern Anal. Machine Intell., vol. 29, no. 4, pp. 640-649, Apr. 2007.

[6]. A.Mian, M. Bennamoun, and R. Owens, "Keypoint detection and local feature matching for textured 3D face recognition,” Int. J. Computer Vision, vol. 79, no. 1, pp. 1-12, 2008.

[7]. T. Theoharis, G. Passalis, G. Toderici, and I. Kakadiaris, "Unified 3D face and ear recognition using wavelets on geometry images," Pattern Recognition, vol. 41, no. 3, pp. 796-804, Mar. 2008.

[8]. P. Yan and K. Bowyer, "Empirical evaluation of advanced ear biometrics," in Proc. CVPR 2005Workshops, Jan. 2005, pp. 41-41. 